\title{
Manejo de lito difícil
}

\author{
Jony Cerna-Cardona* \\ Unidad de Endoscopia, Hospital Juárez de México, Ciudad de México, México
}

\begin{abstract}
Resumen
La colangiografía endoscópica actualmente es la primera línea de tratamiento para la extracción de litos del interior del conducto biliar principal, mediante esfinterotomía y barridos con catéter de balón. Sin embargo, hay litos difíciles (mayores a $1.5 \mathrm{~cm}$, impactados, asociados a conducto biliares más delgados o a estenosis, litos intrahepáticos) que no pueden extraerse con estos métodos convencionales. En la actualidad la dilatación con balón y la litotricia con láser o electrohidráulica se han convertido en herramientas valiosas para la resolución de este tipo de cálculos con una alta tasa de éxito técnico y clínico.
\end{abstract}

Palabras clave: Lito difícil. Dilatación con balón. Colangioscopia por vía oral.

\section{Introducción}

La colangiografía endoscópica (CPE) se considera la primera línea de tratamiento para la coledocolitiasis mediante las técnicas convencionales de esfinterotomía, extracción de litos con balón y cada vez más en desuso la litotricia mecánica con canastilla (principalmente en EE.UU.). La tasa de resolución de coledocolitiasis con técnicas convencionales se estima en un $85-90 \%$, sin embargo, un $10-15 \%$ de los litos son considerados como litos difíciles y es necesario utilizar otras modalidades de tratamiento para permitir resolución completa de estos ${ }^{1-3}$.

El lito difícil se define como aquel que presenta una o más de las siguientes características: factores del lito (mayor $15 \mathrm{~mm}$ de diámetro, largos, múltiples, intrahepáticos), factores del conducto biliar (asociados a estenosis, estrechamientos 0 angulaciones), relación entre el cálculo y el conducto biliar (litos impactados, intrahepáticos, alteraciones anatómicas posquirúrgicas) ${ }^{1,3}$.
En la Digestive Disease Week 2021, realizado de manera virtual, se presentaron un total de 2 conferencias magistrales y 8 trabajos libres en cartel relacionados al manejo del lito difícil. A continuación, se presenta un resumen de los principales trabajos relacionados con el tema.

\section{Dilatación con balón largo de la papila mayor}

Tanto la Sociedad Americana de Endoscopia Gastrointestinal (ASGE) como la Europea de Endoscopia Gastrointestinal (ESGE) recomiendan la dilatación con balón como tratamiento de primera línea en pacientes con lito difícil ${ }^{2,3}$. En la conferencia magistral presentada por el Dr. Stan Branch, se compara los litos difíciles con un barco dentro de una botella: para poder extraer los litos difíciles del interior del conducto biliar, tenemos dos opciones, hacer el lito más pequeño o aumentar el tamaño del orificio papilar. La técnica de dilatación con balón fue descrita por primera vez en el 2003.

\section{Correspondencia:}


Dr. Branch sugiere que previo a la dilatación con balón de la papila mayor se realice esfinterotomía parcial (1/2 o 1/3 de la esfinterotomía convencional) y posteriormente la dilatación (esta debe de coincidir con el diámetro máximo del conducto biliar o $2 \mathrm{~mm}$ por arriba de este, con una duración de 30 segundos), permitiendo resolución de litos difíciles con una tasa de hemorragia similar al de la esfinterotomía. Los diámetros de los balones progresivos van desde 8-20 mm de diámetro y 5 centímetros de longitud ${ }^{4}$. En el estudio prospectivo multicéntrico de Alburquerque, et al., en donde se comparó la eficacia de la CPE en el lito difícil utilizando dilatación con balón con prótesis $(\mathrm{DB}+\mathrm{P})$ vs. colocación de prótesis biliar (CP). De 1,615 pacientes no se pudo resolver la coledocolitiasis en el 6.6\% (107 pacientes). De los 107 pacientes 97 fueron sometidos a CPE posterior (49/97 dilatación con balón más prótesis y 48/97 colocación de prótesis). Después de la CPE, no se logró resolución en 28/97 (28.8\%). La tasa de resolución de la DB+P del 81.6 vs. $60.4 \%$ en CP (OR: 2.91; IC 95\%: 1.15-7.35; $p=0.026)$, sin diferencia estadística en la tasa de complicaciones. Los autores concluyen que la CPE con $\mathrm{DB}+\mathrm{P}$ es superior a la colocación de prótesis sola en la resolución del lito difícil. Esta estrategia debe plantearse siempre en pacientes con litos mayor a $15 \mathrm{~mm}$, más de 10 litos, litos localizados por arriba de estenosis, con alteraciones biliares anatómicas posquirúrgicas y diámetro del conducto biliar principal mayor a $13 \mathrm{~mm}^{5}$.

\section{Colangioscopia peroral y litotricia con láser o electrohidráulica}

Se considera la primera línea de tratamiento en litos intrahepáticos, litos impactados y litos asociados a estenosis o conductos distales adelgazados con relación con el tamaño del lito ${ }^{2,3}$. Un metaanálisis reciente demostró una tasa de éxito de fragmentación de litos del $91.2 \%$ con una resolución completa de la coledocolitiasis en una sola sesión del $76.9 \%$. No existe diferencia estadística de resolución si se compara láser vs. electrohidráulica (92.9 vs. $90.1 \% ; p=0.360)^{6}$. McDonald, et al. utilizaron en un estudio retrospectivo unicéntrico la colangioscopia anterógrada percutánea para el manejo de la enfermedad biliar en pacientes con anatomía alterada quirúrgicamente, un total de 7 pacientes fueron incluidos, la indicación más frecuente fue la coledocolitiasis y colangitis (71-4\%). En todos los casos se logró el éxito técnico y clínico. Estos datos sugieren que este enfoque es seguro, sencillo y solo requiere experiencia en colangioscopia?.

\section{Anatomía alterada posquirúrgica}

La resolución de la coledocolitiasis en el contexto de anatomía alterada posquirúrgica (bypass gástrico en $Y$ de Roux, Billroth II) es un reto para el endoscopista de la vía biliar. En un estudio retrospectivo unicéntrico se evaluaron los resultados de la CPE asistida por laparoscopia (CPE-LP) vs. el drenaje biliar percutáneo (DBP) en pacientes con antecedente de bypass gástrico en $Y$ de Roux de abril 2015 a octubre 2020. Se identificaron 45 pacientes con anatomía alterada en $Y$ de Roux, 30 fueron sometidos a CPE-LP y 15 a DBP. La principal indicación en el grupo CPE-LP fue la coledocolitiasis (27/30, 90\%), mientras que en el grupo DBP fue la estenosis maligna en el $47 \%$, seguido de la coledocolitiasis en el $40 \%$. El éxito técnico fue del $93 \%$ (28/30) en el grupo CPE-LP y $100 \%$ en el de DBP $(p=0.55)$. La tasa de éxito clínico fue del 100\% (28/28) en el grupo CPE-LP vs. 93\% (14/15) en el de DBP $(p=0.35)$. El número de reintervenciones y eventos adversos fue mayor en el grupo DBP $(p<0.001$ y $p=0.059$ respectivamente). Los autores concluyen que ambos procedimientos tienen altas tasas de éxito técnico, sin embargo, la CPE-LP requiere menos procedimientos repetidos y se asocia a menor tasa de complicaciones relacionadas con el procedimiento ${ }^{8}$.

En otro estudio retrospectivo, Ishikawa, et al. evaluaron la eficacia y seguridad de la CPE asistida con enteroscopio de doble balón y el uso de dilatación papilar con balón grande (CPE-EDB+DBG) vs. dilatación endoscópica convencional, en pacientes con anatomía alterada quirúrgicamente. Un total de 53 procedimientos de CPE-EDB+DBG fueron realizados en 49 pacientes de febrero 2013 a noviembre del 2020. De 49 pacientes, $12(24.5 \%)$ se sometieron a gastrectomía Billroth II, $22(44.9 \%)$ se sometieron a reconstrucción en $Y$ de Roux (R-Y) con gastrectomía total y 15 (30.6\%) se sometieron a R-Y con gastrectomía parcial. La tasa de éxito general para llegar a la papila fue del $93.9 \%$ (46/49) y la mediana del tiempo para llegar a la papila fue de 14.5 min (IQR: 8.75-25.25). En el 91.3\% se logró una canulación biliar exitosa, la tasa de resolución de coledocolitiasis fue del $97.4 \%$. No hubo diferencia significativa en la tasa de aclaramiento de litos en ambos grupos, pero sí hubo más complicaciones en el grupo de dilatación convencional (29.4 vs. $4.5 \% ; p=0.046$ ). Los autores concluyen que la CPE-DB en pacientes con anatomía alterada quirúrgica tiene altas tasas de éxito técnico y clínico, tasas aceptables de complicaciones para la resolución de la coledocolitiasis, especialmente en pacientes con litos grandes y múltiples ${ }^{9}$. 


\section{Conclusiones}

El lito difícil es un reto para el endoscopista de la vía biliar. El conocer las diferentes herramientas terapéuticas disponibles y aplicación de estas, de manera individual de acuerdo con el caso del paciente, permitirá mayor tasa de éxito técnico y clínico, con reducción significativa de las complicaciones.

\section{Financiamiento}

La presente investigación no ha recibido ayudas específicas provenientes de agencias del sector público, sector comercial o entidades sin ánimo de lucro.

\section{Conflicto de intereses}

Proctor Boston Scientific.

\section{Bibliografía}

1. Angsuwatcharakon $P$, Rerknimitr R. Cracking difficult biliary stones. Clin Endosc. 2021 Mar 16. doi: 10.5946/ce.2020.256-IDEN. Online ahead of print.
2. ASGE Standards of Practice Committee; Buxbaum J, Abbas S, Sultan S, Fishman DS, Qumseya BJ, Cortessis VK, et al. ASGE guideline on the role of endoscopy in the evaluation and management of choledocholithiasis. Gastrointest Endosc. 2019;89:1075-105.

3. Manes G, Paspatis G, Aabakken L, Anderloni A, Arvanitakis M, Ah-Soune $P$, et al. Endoscopic management of common bile duct stones: European Society of Gastrointestinal Endoscopy (ESGE) guideline. Endoscopy. 2019;51(5):472-91.

4. Branch S. Conferencia magistral "Large and intrahepatic duct Stone management between a rock and hard place". Challenges in ERCP. ASGE. DDW 2021.

5. Alburqueque M, Zaragoza N, Miguel I, Figa M, Pijoan E, Miñana JM, et al. Efficacy of ERCP for difficult bile duct Stone clearence: endoscopic papillary balloon dilation with stent vs. stent. Endoscopy [Internet]. 2021;53(S 01):S78. Disponible en: https://www.thieme-connect.com/products/ejournals/abstract/10.1055/s-0041-1724446

6. McCarty TR, Gulati R, Rustagi T. Efficacy and safety of peroral cholangioscopy with intraductal lithotripsy for difficult biliary stones: a systematic review and meta-analysis. Endoscopy. 2021;53(2):110-22.

7. McDonald N, Azeen N, Trikudanathan G, Mallery JS, Freeman ML, Amateau SK. ID: 3522338 Percutaneous antegrade cholangioscopy for management of biliary disease in surgically altered anatomy with inaccessible biliary tree. Gastrointest Endosc [Internet]. 2021;93(6 Suppl):AB136-AB137. Disponible en: https://www.giejournal.org/article/ S0016-5107(21)01202-5/abstract

8. Pleasant T, Jiang D, Pawa S, Powell MS, Jahann DA, Pawa R. ID: 3520457 Outcomes of laparoscopic-assisted endoscopic retrograde cholangiopancreatography vs. percutaneous transhepatic biliary drainage in patients with Roux-en Y gastric bypass surgery. Gastrointest Endosc [Internet]. 2021;93(6 Suppl):AB136-AB137. Disponible en: https://www. giejournal.org/article/S0016-5107(21)01223-2/abstract

9. Ishikawa $T$, Kawashima H, Ohno E, lida T, Suzuki H, Uetsiki K, et al. ID: 3521057 Efficacy and safety of double-balloon endoscopy-assisted endoscopic papillary large balloon dilation for common bile duct stone removal in patients with surgically altered anatomy having an intact papilla. Gastrointest Endosc [Internet]. 2021;93(6 Suppl):AB146-AB147. Disponible en: https://www.giejournal.org/article/S0016-5107(21)01221-9/abstract 